\title{
Crystal structure of $1^{10}$-methoxy-6-thia-1(1,8)-anthracena- 4,8(1,3)-dibenzacyclodeca-2,9-diynaphane, $\mathrm{C}_{33} \mathrm{H}_{22} \mathrm{OS}$
}

\section{G. T. Crisp, P. D. Turner and E. R. T. Tiekink*}

The University of Adelaide, Department of Chemistry, Australia 5005

Received June 4, 1999, CCDC-No. 1267/203

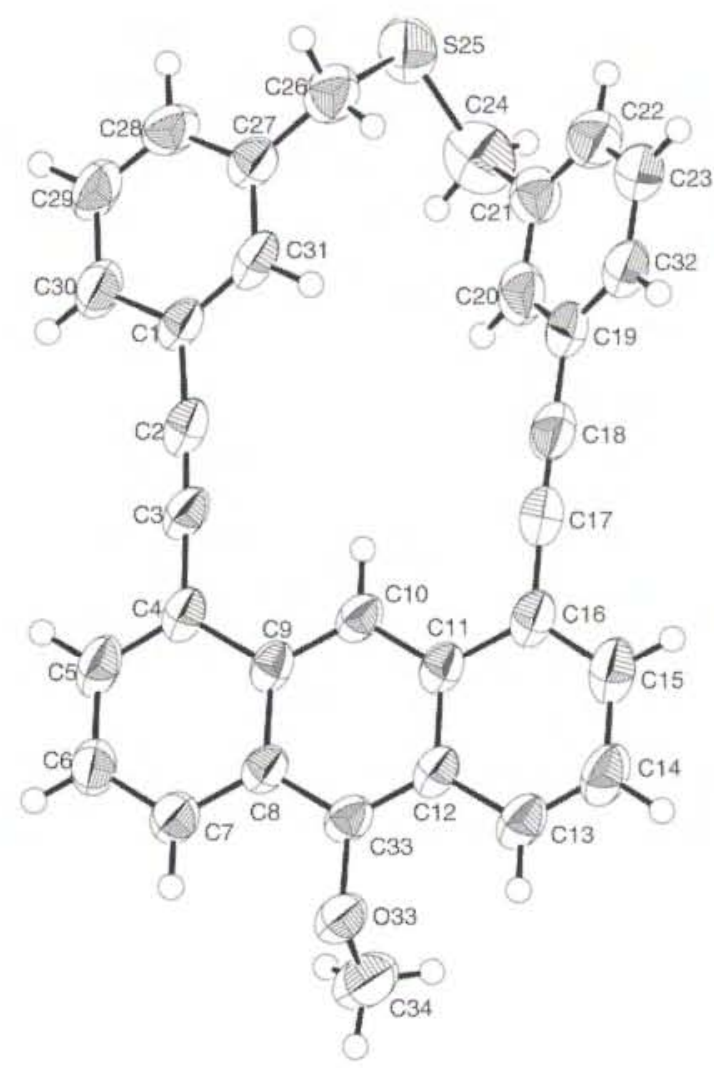

\footnotetext{
Abstract

$\mathrm{C}_{33} \mathrm{H}_{22} \mathrm{OS}$, triclinic, $P \overline{\mathrm{I}}($ No. 2$), a=11.504(7) \AA$,

$b=11.621(8) \AA, c=10.337(8) \AA, \alpha=95.46(6)^{\circ}, \beta=115.96(5)^{\circ}$, $\gamma=98.48(5)^{\circ}, V=1209.0 \AA^{3}, Z=2, R_{\mathrm{gt}}(F)=0.075$, $w R(F)=0.088, T=293 \mathrm{~K}$.
}

\section{Discussion}

Little delocalisation of $\pi$-electron density throughout the molecule is noted as evidenced by the magnitude of the acetylenic $\mathrm{C} 2-\mathrm{C} 3$ and $\mathrm{C} 17-\mathrm{C} 18$ bond distances of $1.197(7) \AA$ and $1.193(7) \AA$, respectively. Further, the molecule is not planar as seen in the dihedral angles formed between the anthracene moiety

and the $\mathrm{C} 1, \mathrm{C} 27-\mathrm{C} 31$ and $\mathrm{C} 19-\mathrm{C} 23, \mathrm{C} 32$ six-membered rings of $12.0^{\circ}$ and $38.9^{\circ}$, respectively. In the lattice, centrosymmetrically related molecules associate via $\pi-\pi$ interactions (closest contact of 3.433(7) $\AA$ occurs between the C27 and C33) atoms and in addition via close $\mathrm{O} 33 \cdots \mathrm{H} 26 \mathrm{~b}^{\prime}$ contacts of $2.77 \AA$ such that $d\left(\mathrm{O} 33 \cdots \mathrm{C} 26^{\prime}\right)$ are 3.491(7) $\dot{\mathrm{A}}$.

Table 1. Data collection and handling.

Crystal:

Wavelength

$\mu$ :

Diffractometer, scan mode:

$20_{\max }$ :

$N(h k l)_{\text {measured, }} N(h k l)_{\text {unique: }}$

Criterion for $I_{\mathrm{obs}}, N(h k l)_{\mathrm{g}}$ :

$N(\text { param })_{\text {refined: }}$

Programs:

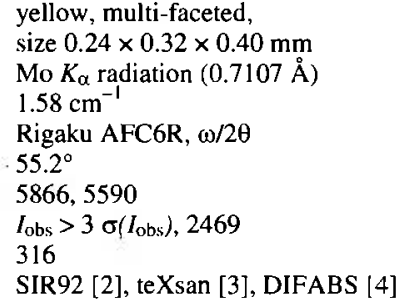

Table 2. Atomic coordinates and displacement parameters (in $\hat{A}^{\hat{z}}$ ).

\begin{tabular}{llllll}
\hline Atom & Site & $x$ & $y$ & $z$ & $U_{\text {isio }}$ \\
\hline $\mathrm{H}(5)$ & $2 i$ & -0.2221 & 0.5116 & -0.2657 & 0.075 \\
$\mathrm{H}(6)$ & $2 i$ & -0.1495 & 0.6589 & -0.0625 & 0.073 \\
$\mathrm{H}(7)$ & $2 i$ & 0.0728 & 0.7464 & 0.07 & 0.067 \\
$\mathrm{H}(10)$ & $2 i$ & 0.1561 & 0.4621 & -0.3006 & 0.057 \\
$\mathrm{H}(13)$ & $2 i$ & 0.532 & 0.7945 & 0.1319 & 0.068 \\
$\mathrm{H}(14)$ & $2 i$ & 0.679 & 0.7323 & 0.0668 & 0.077 \\
$\mathrm{H}(15)$ & $2 i$ & 0.6088 & 0.5856 & -0.1429 & 0.081 \\
$\mathrm{H}(20)$ & $2 i$ & 0.1827 & 0.1741 & -0.4617 & 0.078 \\
$\mathrm{H}(22)$ & $2 i$ & 0.2262 & 0.0022 & -0.787 & 0.083 \\
$\mathrm{H}(23)$ & $2 i$ & 0.3721 & 0.1676 & -0.7794 & 0.081 \\
$\mathrm{H}(24 \mathrm{a})$ & $2 i$ & 0.0458 & -0.009 & -0.5797 & 0.105 \\
$\mathrm{H}(24 \mathrm{~b})$ & $2 i$ & 0.1208 & -0.0971 & -0.6159 & 0.105 \\
$\mathrm{H}(26 \mathrm{a})$ & $2 i$ & -0.0331 & 0.1203 & -0.8321 & 0.082 \\
$\mathrm{H}(26 \mathrm{~b})$ & $2 i$ & -0.1654 & 0.0516 & -0.962 & 0.082 \\
$\mathrm{H}(28)$ & $2 i$ & -0.3501 & -0.0333 & -0.8938 & 0.076 \\
$\mathrm{H}(29)$ & $2 i$ & -0.4695 & 0.0328 & -0.7892 & 0.087 \\
$\mathrm{H}(30)$ & $2 i$ & -0.3757 & 0.1913 & -0.5921 & 0.076 \\
$\mathrm{H}(31)$ & $2 i$ & -0.0408 & 0.2351 & -0.6395 & 0.064 \\
$\mathrm{H}(32)$ & $2 i$ & 0.4321 & 0.3342 & -0.6019 & 0.075 \\
$\mathrm{H}(34 \mathrm{a})$ & $2 i$ & 0.2889 & 0.7171 & 0.2736 & 0.099 \\
$\mathrm{H}(34 \mathrm{~b})$ & $2 i$ & 0.4328 & 0.7401 & 0.2944 & 0.099 \\
$\mathrm{H}(34 \mathrm{c})$ & $2 i$ & 0.3787 & 0.8424 & 0.3412 & 0.099 \\
& & & & & \\
\hline & & & & &
\end{tabular}


Table 3. Atomic coordinates and displacement parameters (in $\AA^{2}$ ).

\begin{tabular}{|c|c|c|c|c|c|c|c|c|c|c|}
\hline Atom & Site & $x$ & $y$ & $z$ & $U_{11}$ & $U_{22}$ & $U_{33}$ & $U_{12}$ & $U_{13}$ & $U_{23}$ \\
\hline$S(25)$ & $2 i$ & $-0.0550(2)$ & $-0.0778(1)$ & $-0.8272(2)$ & $0.068(1)$ & $0.047(1)$ & $0.131(2)$ & $0.0075(8)$ & $0.039(1)$ & $-0.010(1)$ \\
\hline$O(33)$ & $2 i$ & $0.3129(3)$ & $0.8019(3)$ & $0.1327(4)$ & $0.047(2)$ & $0.049(2)$ & $0.052(2)$ & $0.007(2)$ & $0.009(2)$ & $0.003(2)$ \\
\hline $\mathrm{C}(1)$ & $2 i$ & $-0.1970(5)$ & $0.2315(4)$ & $-0.5984(5)$ & $0.035(3)$ & $0.041(3)$ & $0.052(3)$ & $0.008(2)$ & $0.005(3)$ & $0.009(3)$ \\
\hline $\mathrm{C}(2)$ & $2 i$ & $-0.1376(5)$ & $0.3310(5)$ & $-0.4784(6)$ & $0.033(3)$ & $0.050(4)$ & $0.061(4)$ & $0.008(3)$ & $0.011(3)$ & $0.017(3)$ \\
\hline $\mathrm{C}(3)$ & $2 i$ & $-0.0939(5)$ & $0.4110(4)$ & $-0.3768(6)$ & $0.033(3)$ & $0.039(3)$ & $0.057(3)$ & $0.002(2)$ & $0.005(3)$ & $0.004(3)$ \\
\hline $\mathrm{C}(4)$ & $2 i$ & $-0.0459(5)$ & $0.5038(4)$ & $-0.2565(6)$ & $0.032(3)$ & $0.044(3)$ & $0.056(3)$ & $0.005(2)$ & $0.012(3)$ & $0.012(3)$ \\
\hline$C(5)$ & $2 i$ & $-0.1303(5)$ & $0.5449(5)$ & $-0.2118(7)$ & $0.032(3)$ & $0.051(4)$ & $0.084(4)$ & $0.002(3)$ & $0.012(3)$ & $0.008(3)$ \\
\hline$C(6)$ & $2 i$ & $-0.0873(5)$ & $0.6345(5)$ & $-0.0906(6)$ & $0.040(3)$ & $0.055(4)$ & $0.076(4)$ & $0.010(3)$ & $0.019(3)$ & $0.000(3)$ \\
\hline$C(7)$ & $2 i$ & $0.0437(5)$ & $0.6862(4)$ & $-0.0130(6)$ & $0.046(3)$ & $0.043(3)$ & $0.063(4)$ & $0.013(3)$ & $0.012(3)$ & $0.001(3)$ \\
\hline $\mathrm{C}(8)$ & $2 i$ & $0.1373(5)$ & $0.6513(4)$ & $-0.0544(5)$ & $0.038(3)$ & $0.037(3)$ & $0.046(3)$ & $0.007(2)$ & $0.009(3)$ & $0.007(2)$ \\
\hline$C(9)$ & $2 i$ & $0.0953(5)$ & $0.5590(4)$ & $-0.1769(5)$ & $0.034(3)$ & $0.038(3)$ & $0.052(3)$ & $0.008(2)$ & $0.010(2)$ & $0.013(3)$ \\
\hline$C(10)$ & $2 i$ & $0.1856(5)$ & $0.5248(4)$ & $-0.2199(5)$ & $0.039(3)$ & $0.037(3)$ & $0.050(3)$ & $0.003(2)$ & $0.007(3)$ & $0.003(2)$ \\
\hline$C(11)$ & $2 i$ & $0.3200(5)$ & $0.5815(4)$ & $-0.1456(5)$ & $0.036(3)$ & $0.042(3)$ & $0.052(3)$ & $0.009(2)$ & $0.011(3)$ & $0.012(3)$ \\
\hline$C(12)$ & $2 i$ & $0.3643(5)$ & $0.6742(4)$ & $-0.0215(5)$ & $0.035(3)$ & $0.043(3)$ & $0.050(3)$ & $0.004(2)$ & $0.010(3)$ & $0.010(3)$ \\
\hline$C(13)$ & $2 i$ & $0.5018(5)$ & $0.7307(5)$ & $0.0522(6)$ & $0.040(3)$ & $0.046(3)$ & $0.063(4)$ & $0.000(3)$ & $0.010(3)$ & $0.007(3)$ \\
\hline$C(14)$ & $2 i$ & $0.5879(5)$ & $0.6965(5)$ & $0.0119(7)$ & $0.036(3)$ & $0.060(4)$ & $0.080(4)$ & $-0.004(3)$ & $0.017(3)$ & $0.007(3)$ \\
\hline$C(15)$ & $2 i$ & $0.5460(5)$ & $0.6059(5)$ & $-0.1132(7)$ & $0.039(3)$ & $0.064(4)$ & $0.091(5)$ & $0.011(3)$ & $0.023(3)$ & $0.019(4)$ \\
\hline$C(16)$ & $2 i$ & $0.4147(5)$ & $0.5485(5)$ & $-0.1906(6)$ & $0.037(3)$ & $0.051(3)$ & $0.062(4)$ & $0.010(3)$ & $0.012(3)$ & $0.013(3)$ \\
\hline$C(17)$ & $2 i$ & $0.3755(5)$ & $0.4560(5)$ & $-0.3101(7)$ & $0.038(3)$ & $0.062(4)$ & $0.064(4)$ & $0.014(3)$ & $0.020(3)$ & $0.019(3)$ \\
\hline$C(18)$ & $2 i$ & $0.3470(5)$ & $0.3760(6)$ & $-0.4072(7)$ & $0.042(4)$ & $0.072(4)$ & $0.067(4)$ & $0.020(3)$ & $0.011(3)$ & $0.008(4)$ \\
\hline$C(19)$ & $2 i$ & $0.3118(5)$ & $0.2746(5)$ & $-0.5192(6)$ & $0.039(3)$ & $0.072(4)$ & $0.053(4)$ & $0.023(3)$ & $0.012(3)$ & $0.013(3)$ \\
\hline$C(20)$ & $2 i$ & $0.2202(6)$ & $0.1738(6)$ & $-0.5276(6)$ & $0.051(4)$ & $0.084(5)$ & $0.063(4)$ & $0.033(3)$ & $0.021(3)$ & $0.025(4)$ \\
\hline$C(21)$ & $2 i$ & $0.1866(6)$ & $0.0735(5)$ & $-0.6338(6)$ & $0.057(4)$ & $0.065(4)$ & $0.056(4)$ & $0.021(3)$ & $0.023(3)$ & $0.019(3)$ \\
\hline $\mathrm{C}(22)$ & $2 i$ & $0.2458(6)$ & $0.0724(5)$ & $-0.7198(7)$ & $0.060(4)$ & $0.065(4)$ & $0.070(4)$ & $0.016(3)$ & $0.019(3)$ & $0.005(3)$ \\
\hline$C(23)$ & $2 i$ & $0.3348(6)$ & $0.1691(6)$ & $-0.7134(6)$ & $0.048(4)$ & $0.088(5)$ & $0.062(4)$ & $0.017(3)$ & $0.021(3)$ & $0.006(4)$ \\
\hline$C(24)$ & $2 i$ & $0.0802(7)$ & $-0.0321(6)$ & $-0.6446(8)$ & $0.116(6)$ & $0.072(5)$ & $0.094(5)$ & $0.040(4)$ & $0.053(5)$ & $0.040(4)$ \\
\hline$C(26)$ & $2 i$ & $-0.1094(6)$ & $0.0598(5)$ & $-0.8603(6)$ & $0.060(4)$ & $0.063(4)$ & $0.068(4)$ & $0.020(3)$ & $0.014(3)$ & $0.009(3)$ \\
\hline$C(27)$ & $2 i$ & $-0.1823(5)$ & $0.0978(4)$ & $-0.7816(6)$ & $0.045(3)$ & $0.043(3)$ & $0.052(3)$ & $0.009(3)$ & $0.011(3)$ & $0.008(3)$ \\
\hline$C(28)$ & $2 i$ & $-0.3117(6)$ & $0.0361(5)$ & $-0.8206(6)$ & $0.056(4)$ & $0.048(4)$ & $0.064(4)$ & $0.001(3)$ & $0.014(3)$ & $-0.006(3)$ \\
\hline$C(29)$ & $2 i$ & $-0.3807(5)$ & $0.0729(5)$ & $-0.7556(7)$ & $0.037(4)$ & $0.060(4)$ & $0.090(5)$ & $-0.004(3)$ & $0.012(3)$ & $-0.008(4)$ \\
\hline$C(30)$ & $2 i$ & $-0.3267(5)$ & $0.1690(5)$ & $-0.6408(6)$ & $0.038(3)$ & $0.064(4)$ & $0.070(4)$ & $0.007(3)$ & $0.012(3)$ & $0.004(3)$ \\
\hline$C(31)$ & $2 i$ & $-0.1285(5)$ & $0.1936(4)$ & $-0.6696(6)$ & $0.031(3)$ & $0.039(3)$ & $0.066(4)$ & $0.003(2)$ & $0.002(3)$ & $0.013(3)$ \\
\hline$C(32)$ & $2 i$ & $0.3679(5)$ & $0.2687(5)$ & $-0.6101(6)$ & $0.038(3)$ & $0.077(4)$ & $0.061(4)$ & $0.011(3)$ & $0.015(3)$ & $0.009(3)$ \\
\hline$C(33)$ & $2 i$ & $0.2723(5)$ & $0.7068(4)$ & $0.0193(5)$ & $0.042(3)$ & $0.035(3)$ & $0.050(3)$ & $0.006(2)$ & $0.008(3)$ & $0.002(3)$ \\
\hline$C(34)$ & $2 i$ & $0.3567(6)$ & $0.7729(6)$ & $0.2716(7)$ & $0.076(5)$ & $0.074(5)$ & $0.064(4)$ & $0.014(4)$ & $0.006(4)$ & $0.001(4)$ \\
\hline
\end{tabular}

Acknowledgment. The Australian Research Council is thanked for the support.

\section{References}

1. Bodwell, G. J.; Houghton, T. J.; Koury, H. E.; Yarlagadda, B.: Sodium sulfide adsorbed on alumina as a reagent for the facile synthesis of 2,11-dithia[3.3]metacyclophanes. Synlett (1995) 751-752.
2. Altomare, A.; Cascarano, M.; Giacovazzo, C.; Guagliardi, A.: SIR92 - a program for automatic solution of crystal structures by direct methods. J. Appl. Crystallogr. 26 (1993) 343.

3. Molecular Structure Corporation. (1997-1998). teXsan for Windows version 1.03. Single Crystal Structure Analysis Software. Version 1.04. MSC, 3200 Research Forest Drive, The Woodlands, TX 77381, USA.

4. Walker, N.; Stuart, D.: An empirical method for correcting diffractometer data for absorption effects. Acta Crystallogr. A39 (1983) 159-166. 